\title{
Infecções da corrente sanguínea em unidade de terapia intensiva: estudo retrospectivo em um hospital de ensino
}

\author{
Bloodstream infections in intensive care unit: retrospective \\ study in a teaching hospital
}

\author{
Betina Brixner ${ }^{\bullet}$ Nayanna Dias Bierhals ${ }^{2} \bullet$ Caio Fernando de Oliveira ${ }^{3} \bullet$ Jane Dagmar Pollo Renner ${ }^{4}$
}

\begin{abstract}
RESUMO
O objetivo deste estudo foi verificar os fatores de risco e perfil epidemiológico dos pacientes diagnosticados com infecção de corrente sanguínea, bem como os microrganismos responsáveis pela infecção. Estudo transversal, em que foi realizado um levantamento das hemoculturas e dados dos pacientes internados em unidade de terapia intensiva adulto com diagnóstico de infecção de corrente sanguínea, durante o ano de 2016. Foram coletadas informações referente ao paciente e sua internação, bem como ao agente responsável pela infecção e seu perfil de resistência. Foram incluídas 24 hemoculturas positivas para crescimento bacteriano. A média de idade dos pacientes foi de $53,9 \pm 2$ I, I anos e $54,5 \%$ dos pacientes acometidos pela infecção eram homens. Dos pacientes, 59,1\% apresentavam histórico de doença cardíaca, sendo que destes, $63,6 \%$ foram a óbito. As bactérias Gram positivas foram mais relacionadas com a infecção, em que 54,2\% eram Staphylococcus coagulase negativa e destes, $76,9 \%$ foram resistentes meticilina. Identificou-se que o sexo masculino, indivíduos idosos e com histórico de alguma comorbidades prévia, com destaque para as doenças cardíacas, foram os mais acometidos com bacteremia. Quanto ao agente bacteriano responsável pela infecção, o Staphylococcus coagulase negativa foi o mais relacionado aos casos diagnosticados, bem como o seu alto perfil de resistência deste microrganismo frente a meticilina. Palavras-chave: Bacteremia; Fatores de Risco; Diagnóstico; Unidade de Terapia Intensiva.
\end{abstract}

\begin{abstract}
The objective of this study was to verify the risk factors and epidemiological profile of the patients diagnosed with bloodstream infection, as well as the microorganisms responsible for the infection. A cross-sectional study was carried out in which blood cultures were collected and data were collected from patients admitted to an adult intensive care unit with a diagnosis of bloodstream infection during the year 2016. Information about the patient and hospitalization was collected, as well as the agent responsible for the infection 'and its resistance profile. 24 blood cultures positive for bacterial growth were included. The mean age of the patients was $53.9 \pm 21$.I years and $54.5 \%$ of the patients affected by the infection were men. Of the patients, $59.1 \%$ had a history of heart disease, of which $63.6 \%$ died. Gram positive bacteria were more related to infection, in which $54.2 \%$ were coagulase negative Staphylococcus and of these, $76.9 \%$ were resistant to methicillin. It was identified that the male sex, elderly individuals and with history of some previous comorbidities, especially heart diseases, were the most affected with bacteremia. As for the bacterial agent responsible for the infection, Coagulase negative Staphylococcus was the most related to the diagnosed cases, as well as its high resistance profile of this microorganism against methicillin.
\end{abstract}

Keywords: Bacteremia; Risk factors; Diagnosis; Intensive Care Unit.

\footnotetext{
'Farmacêutica. Mestra em Promoção da Saúde pela Universidade de Santa Cruz do Sul (UNISC). RS, Brasil. E-mail: betinabrixner@yahoo.com.br ${ }^{2}$ Acadêmica do Curso de Farmácia e bolsista de iniciação científica na Universidade de Santa Cruz do Sul (UNISC). RS, Brasil. E-mail: nayanna.db@outlook.com ${ }^{3}$ Farmacêutico bioquímico. Pós-doutorando do programa de pós graduação em Promoção da Saúde da Universidade de Santa Cruz do Sul (UNISC). RS, Brasil. E-mail: caiofarm@yahoo.com.br

${ }^{4}$ Farmacêutica Bioquímica. Doutora em Biologia Celular e Molecular pela Pontifícia Universidade Católica do Rio Grande do Sul (PUCRS). Docente do departamento de Biologia e Farmácia e do programa de pós graduação em Promoção da Saúde na Universidade de Santa Cruz do Sul (UNISC). RS, Brasil. E-mail: janerenner@unisc.br
} 


\section{INTRODUÇÃO}

Dentre as Infecções Relacionadas a Assistência à Saúde (IRAS) que podem acometer um paciente em estado crítico, a infecção da corrente sanguínea (ICS) está entre uma das mais frequentes ${ }^{(1-3)}$. Este sítio está classificado como o terceiro mais acometido por infecções nas Unidades de Terapia Intensiva (UTI), tanto a nível mundial quanto Brasil, sendo responsável por causar, respectivamente, I5, I\% e 10,1\% das IRAS nesta unidade de internação(1,2).

Considerada uma doença infecciosa grave, em decorrência da invasão de microrganismos no sangue circulante e com risco de carrear microrganismos para outros órgãos, a ICS é responsável por causar desfechos clínicos desfavoráveis ${ }^{(4,5)}$.Além da morbimortalidade, esta infecção também está associada com o aumento do tempo de internação e com elevados gastos de procedimentos diagnósticos e medicamentosos ${ }^{(6)}$.

O diagnóstico de ICS é desafiador, em que a detecção, identificação microbiana e resultado dos testes de susceptibilidade são de extrema importância para o início de uma antibioticoterapia adequada, pois quanto mais precoce o seu início, melhor será o prognóstico e as chances de sobrevida do paciente ${ }^{(4)}$.

Em virtude deste problema de saúde pública, o presente estudo teve como objetivo traçar um perfil epidemiológico dos pacientes acometidos por ICS, bem como dos microrganismos responsáveis por causar esta infecção.

\section{MÉTODOS}

Trata-se de um estudo retrospectivo de caráter transversal, realizado através do levantamento de ICS em pacientes internados em UTI adulto, durante o período de janeiro a dezembro de 2016, em um hospital de ensino do interior do Rio Grande do Sul - RS, Brasil. Este é um hospital filantrópico e de grande porte, com 232 leitos disponíveis, em que dez destes são destinados à UTI adulto. Cabe ressaltar que esta UTI atende pacientes clínicos e cirúrgicos, porém atende em maior número internações de origem clínica. Também é considerado referência para atendimentos de alta complexidade nas áreas de ortopedia e traumatologia e, também, em atendimentos para gestantes de alto risco.

Para selecionar as amostras utilizadas neste estudo, houve auxílio do laboratório de análises clínicas, localizado juntamente ao hospital, o qual gerou e disponibilizou um relatório de todas as hemoculturas realizadas no período do estudo. Posteriormente, identificou-se as hemoculturas que eram provenientes de pacientes internados na UTI adulto, selecionando somente aquelas com resultado positivo para crescimento bacteriano.
Após, foi realizada uma busca ativa no sistema informatizado (MV 2000 ${ }^{\circledR}$ ) do hospital, utilizando um questionário para o preenchimento de informações referente ao paciente e sua internação, bem como ao agente patogênico responsável pela ICS e seu perfil de resistência aos antimicrobianos.

Cabe ressaltar que as hemoculturas foram realizadas pelo laboratório de análises clínicas, o qual utiliza técnicas automatizadas (Bactec $囚 e$ AutoScan 4 System $\AA$ ) com o propósito de agilizar e qualificar os resultados para os pacientes em estado crítico de saúde.

Como critérios de inclusão para este estudo, tem-se os pacientes com diagnóstico de ICS, de ambos os sexos e com idade superior a 13 anos. Como critérios de exclusão, aqueles pacientes cujos prontuários não estavam completos, impossibilitando a coleta das variáveis e as ICS causadas por fungos.

Os dados foram analisados no SPSS versão 23.0 (SPSS Inc., Armank, NY, USA), em que a estatística descritiva das variáveis, foi avaliada através de frequência, média e desvio padrão.As associações entre as variáveis categóricas, foram avaliadas utilizando o teste de Qui Quadrado, considerando resultado estatisticamente significativo um valor de $p \leq 0,05$.

Este estudo faz parte de um projeto-mãe intitulado "Desenvolvimento de métodos moleculares no diagnóstico de microrganismos e genes envolvidos na resistência bacteriana", o qual foi aprovado pelo Comitê de Ética em Pesquisa da Universidade de Santa Cruz do Sul, sob Parecer de $n^{\circ} 1.540 .110 / 2016$ e CAEE de $n^{\circ}$ $554947 \mid 6.3 .0000 .5343$.

\section{RESULTADOS}

Durante o período do estudo, foram solicitadas 85 hemoculturas automatizadas na UTI adulto, sendo que para cada paciente houve coleta de duas amostras em locais distintos.Após a seleção das amostras, verificou-se que $3 \mathrm{I}$ hemoculturas apresentaram resultados positivos e, após os critérios de exclusão, o estudo permaneceu com um total de 24 amostras.

Analisando os dados, verificou-se que em dois casos houve infecção recorrente de corrente sanguínea, sendo assim, as variáveis populacionais foram calculadas de acordo com a quantidade de pacientes com o diagnóstico da infecção (Tabela I).A idade dos pacientes acometidos pela ICS variou de 14 a 80 anos, cuja média de idade foi de $53,9 \pm 21, I$.

O tempo de permanência destes pacientes na UTI variou de 2 a 38 dias, apresentando uma média de 12,0 $\pm 9,4$ dias. A tabela 2 demostra que entre os pacientes com diagnóstico de ICS, 20 (90,9\%) apresentavam histórico prévio de doenças, sendo que a cardíaca foi encontrada com maior frequência neste estudo. Além 
disso, também é possível visualizar que I4 $(63,6 \%)$ desses pacientes foram a óbito, apresentando resultados estatisticamente significativos quando comparado o número de pacientes com ICS que foram a óbito e que possuíam histórico de doença cardíaca, indicando que esta patologia pode ter relação com os óbitos de pacientes com diagnóstico de ICS.

Das 24 ICS encontradas neste estudo, observou-se que em $23(95,8 \%)$ casos os pacientes apresentavam sinais indicativos de infecção, como febre e/ou hipotensão e, em decorrência destes quadros clínicos, houve a necessidade de confirmar a ICS e seu microrganismo patogênico através de hemocultura automatizada. Verificou-se também, que em 13 (54,2\%) amostras de hemoculturas coletadas, os pacientes estavam fazendo uso de antibioticoterapia prévia para tratamento ou profilaxia.

A Tabela 3 indica os agentes causadores da ICS neste estudo, sendo possível observar a predominância destas infecções por bactéria Gram positivas. O Staphylococcus coagulase negativa ( $\mathrm{SCoN}$ ) foi o microrganismo mais relacionado as ICS nesta UTI, com $13(54,2 \%)$ casos e, destes, 10 (76,9\%) foram considerados Staphylococcus coagulase negativa resistente a meticilina (MRSCoN).

$\mathrm{Na}$ Tabela 4, pode-se observar que a maioria dessas infecções são nosocomiais e, não houve relação entre o uso de dispositivo médico invasivo e a origem da ICS.

\section{DISCUSSÃO}

É de extrema importância avaliar quais são as bactérias envolvidas nos casos de infecções nosocomiais; desta maneira consegue-se evitar situações de surtos de microrganismos multirresistentes e controlar este preocupante problema de saúde pública, devido ao aumento da morbimortalidade e demais fatores que refletem diretamente na segurança do paciente internado ${ }^{(7)}$.

As ICS foram mais prevalentes em pacientes do sexo masculino, corroborando com outros estudos ${ }^{(2,4,6,8)}$. Apesar das IRAS não estarem associadas ao uso de dispositivos médicos invasivos, há dados na literatura mostrando que os homens estão predispostos a desenvolver infecção neste sítio; uma vez que possuem maior risco de colonização bacteriana em sua pele, devendo haver cuidados intensificados na colocação do cateter venoso central e durante sua permanência ${ }^{(8,9)}$.Desta maneira, as taxas elevadas de ICS em indivíduos do sexo masculino estão relacionadas com o maior risco da microbiota da pele e, também, em relação com as ainda desconhecidas distinções anatômicas entre os sexos ${ }^{(8)}$.

Das 24 bacteremias incluídas neste estudo, 17 foram de origem nosocomial, sendo que somente duas estavam associadas ao uso de dispositivo médico invasivo. A utilização de cateteres é considerada um fator de risco potencial para ICS, principalmente para os pacientes internados em UTI, porém, consegue-se reduzir os índices quando se aplica corretamente todas as etapas do bundle de prevenção de infecção relacionada ao uso de cateter ${ }^{(9,10)}$. Ainda houve uma notificação de ICS associada à dispositivo invasivo, de origem comunitária; o paciente fazia uso de cateter urinário pois realizava hemodiálise em clínica médica. $A$ tendência de prevalência de infecção em pacientes em hemodiálise é aumentada, devido ao uso de cateteres venosos centrais ${ }^{(1,2,11)}$. Diante disso, é indispensável educar a equipe multiprofissional e os pacientes, adotar medidas de prevenção que devem ser aplicadas de maneira adequadas em unidades de tratamento de hemodiálise ${ }^{(1,12)}$.

TABELA 1 - Dados dos pacientes com diagnóstico de ICS e tipo de internação, na UTI adulto de um hospital de ensino. Santa Cruz do Sul, RS, Brasil, 2016.

\begin{tabular}{l|c}
\hline VARIÁVEIS & $\mathbf{n}(\%)$ \\
\hline Sexo & $10(45,5)$ \\
\hline Feminino & $12(54,5)$ \\
\hline Masculino & $2(9,1)$ \\
\hline Faixa etária & $3(13,6)$ \\
\hline$\leq 18$ anos & $4(18,2)$ \\
\hline $19-30$ anos & $13(59,1)$ \\
\hline $40-59$ anos & \\
\hline Tipo de internação & $18(81,8)$ \\
\hline Clínica & $2(9,1)$ \\
\hline Cirúrgica eletiva & $2(9,1)$ \\
\hline Cirúrgica de emergência & $22(100,0)$ \\
\hline TOTAL & \\
\hline
\end{tabular}

Fonte: Dados da pesquisa 
TABELA 2 - Relação das patologias prévias dos pacientes com diagnóstico de ICS internados na UTI adulto de um hospital de ensino. Santa Cruz do Sul, RS, Brasil, 2016.

\begin{tabular}{|c|c|c|c|c|c|c|c|c|c|}
\hline \multirow{3}{*}{$\begin{array}{l}\text { HISTÓRICO DE } \\
\text { DOENÇA }\end{array}$} & \multicolumn{4}{|c|}{ COMORBIDADES } & \multicolumn{5}{|c|}{ DESFECHO } \\
\hline & Sim & Não & Total & & Alta & Transferência & Óbito & Total & \\
\hline & n (\%) & n (\%) & n (\%) & & n (\%) & n (\%) & n (\%) & n (\%) & \\
\hline \multicolumn{10}{|l|}{ Cardíaca } \\
\hline Sim & $13(59,1)$ & $0(0)$ & $13(59,1)$ & \multirow{2}{*}{0,075} & $1(4,5)$ & $1(4,5)$ & $11(50,0)$ & $13(59,1)$ & \multirow{2}{*}{0,035} \\
\hline Não & $7(31,8)$ & $2(9,1)$ & $9(40,9)$ & & $5(22,7)$ & $1(4,5)$ & $3(13,6)$ & $9(40,9)$ & \\
\hline \multicolumn{10}{|l|}{ Pulmonar } \\
\hline Sim & $1(4,5)$ & $0(0)$ & $1(4,5)$ & \multirow{2}{*}{0,746} & $0(0)$ & $0(0)$ & $1(4,5)$ & $1(4,5)$ & \multirow{2}{*}{0,741} \\
\hline Não & $19(86,4)$ & $2(9,1)$ & $21(95,5)$ & & $6(27,3)$ & $2(9,1)$ & $13(59,1)$ & $21(95,5)$ & \\
\hline \multicolumn{10}{|l|}{ Neurológica } \\
\hline Sim & $2(9,1)$ & $0(0)$ & $2(9,1)$ & \multirow{2}{*}{0,639} & $1(4,5)$ & $0(0)$ & $1(4,5)$ & $2(9,1)$ & \multirow{2}{*}{0,711} \\
\hline Não & $18(81,8)$ & $2(9,1)$ & $20(90,9)$ & & $5(22,7)$ & $2(9,1)$ & $13(59,1)$ & $20(90,9)$ & \\
\hline \multicolumn{10}{|l|}{ Malignidade } \\
\hline Sim & $1(4,5)$ & $0(0)$ & $1(4,5)$ & \multirow{2}{*}{0,746} & $0(0)$ & $0(0)$ & $1(4,5)$ & $1(4,5)$ & \multirow{2}{*}{0,741} \\
\hline Não & $19(86,4)$ & $2(9,1)$ & $21(95,5)$ & & $6(27,3)$ & $2(9,1)$ & $13(59,1)$ & $21(95,5)$ & \\
\hline \multicolumn{10}{|l|}{ Endocrinológica } \\
\hline Sim & $4(18,2)$ & $0(0)$ & $4(18,2)$ & \multirow{2}{*}{0,484} & $0(0)$ & $1(4,5)$ & $3(13,6)$ & $4(18,2)$ & \multirow{2}{*}{0,247} \\
\hline Não & $16(72,7)$ & $2(9,1)$ & $18(81,8)$ & & $6(27,3)$ & $1(4,5)$ & $11(50)$ & $18(81,8)$ & \\
\hline \multicolumn{10}{|l|}{ Renal aguda } \\
\hline Sim & $1(4,5)$ & $0(0)$ & $1(4,5)$ & \multirow{2}{*}{0,746} & $0(0)$ & $0(0)$ & $1(4,5)$ & $1(4,5)$ & \multirow{2}{*}{0,741} \\
\hline Não & $19(86,4)$ & $2(9,1)$ & $21(95,5)$ & & $6(27,3)$ & $2(9,1)$ & $13(59,1)$ & $21(95,5)$ & \\
\hline \multicolumn{10}{|l|}{ Renal crônica } \\
\hline Sim & $1(4,5)$ & $0(0)$ & $1(4,5)$ & \multirow{2}{*}{0,746} & $0(0)$ & $0(0)$ & $1(4,5)$ & $1(4,5)$ & \multirow{2}{*}{0,741} \\
\hline Não & $19(86,4)$ & $2(9,1)$ & $21(95,5)$ & & $6(27,3)$ & $2(9,1)$ & $13(59,1)$ & $21(95,5)$ & \\
\hline \multicolumn{10}{|l|}{ Hepática } \\
\hline Sim & $1(4,5)$ & $0(0)$ & $1(4,5)$ & \multirow{2}{*}{0,746} & $0(0)$ & $0(0)$ & $1(4,5)$ & $1(4,5)$ & \multirow{2}{*}{0,741} \\
\hline Não & $19(86,4)$ & $2(9,1)$ & $21(95,5)$ & & $6(27,3)$ & $2(9,1)$ & $13(59,1)$ & $21(95,5)$ & \\
\hline \multicolumn{10}{|l|}{ Imunodeficiência } \\
\hline Sim & $3(13,6)$ & $0(0)$ & $3(13,6)$ & \multirow{2}{*}{0,556} & $2(9,1)$ & $1(4,5)$ & $0(0)$ & $3(13,6)$ & \\
\hline Não & $17(77,3)$ & $2(9,1)$ & $19(86,4)$ & & $4(18,2)$ & $1(4,5)$ & $14(63,6)$ & $19(86,4)$ & \\
\hline TOTAL & $20(90,9)$ & $2(9,1)$ & $22(100,0)$ & & $6(27,3)$ & $2(9,1)$ & $14(63,6)$ & $22(100,0)$ & \\
\hline
\end{tabular}

Nota: * Teste do Qui-quadrado de Person.

Fonte: Dados da pesquisa 
TABELA 3 - Microrganismos causadores de ICS e mecanismos de resistência fenotípico encontrado na UTI adulto de um hospital de ensino. Santa Cruz do Sul, RS, Brasil, 2016.

\begin{tabular}{|c|l|c|}
\hline \multicolumn{1}{|c|}{ VARIÁVEIS } & n (\%) \\
\hline \multicolumn{1}{|l|}{ Microrganismo causador da ICS } & $13(54,2)$ \\
\hline & SCoN positivo & $3(12,5)$ \\
\hline & Enterococcus spp. & $2(8,3)$ \\
\hline & Staphylococcus aureus & \\
\hline & Gram negativo & $2(8,3)$ \\
\hline & Klebsiella pneumoniae & $2(8,3)$ \\
\hline & Escherichia coli & $1(4,2)$ \\
\hline & Serratia spp. & $1(4,2)$ \\
\hline Mecanismo de resistência fenotípico & \\
\hline & MRSCoN/MRSA & $10(41,7)$ \\
\hline & ESBL & $2(8,3)$ \\
\hline & Ausente/Não testado & $12(50,0)$ \\
\hline TOTAL & & $24(100,0)$ \\
\hline
\end{tabular}

Nota: SCoN (Staphylococcus coagulase negativa); MRSCoN (Staphylococcus coagulase negativa resistente a meticilina); ESBL ( $\beta$-Lactamase de Espectro Estendido).

Fonte: Dados da pesquisa

TABELA 4 - Relação entre o uso de dispositivo invasivo e origem das ICS em pacientes internados na UTI adulto de um hospital de ensino. Santa Cruz do Sul, RS, Brasil, 2016.

\begin{tabular}{|c|c|c|c|c|}
\hline \multirow{3}{*}{ Origem da Infecção } & \multicolumn{4}{|c|}{ Infecção ligada ao uso de dispositivo invasivo } \\
\hline & \multirow{2}{*}{$\begin{array}{c}\text { Sim } \\
\mathrm{n}(\%)\end{array}$} & \multirow{2}{*}{$\begin{array}{c}\text { Não } \\
n(\%)\end{array}$} & \multirow{2}{*}{\begin{tabular}{|c|} 
Total \\
$\mathbf{n}(\%)$
\end{tabular}} & \multirow{2}{*}{$\mathbf{p}^{*}$} \\
\hline & & & & \\
\hline Comunitária & $1(4,2)$ & $6(25,0)$ & $7(29,2)$ & \multirow{2}{*}{0,875} \\
\hline Nosocomial & $2(8,3)$ & $15(62,5)$ & $17(70,8)$ & \\
\hline TOTAL & $3(12,5)$ & $21(87,5)$ & $24(100)$ & \\
\hline
\end{tabular}

Nota: * Teste do Qui-quadrado de Person.

Fonte: Dados da pesquisa

No presente estudo, 59, $1 \%$ dos pacientes que foram diagnosticados com ICS apresentavam idade igual ou superior a 60 anos. A vulnerabilidade da população geriátrica deixa-os mais propensos a desenvolver bacteremia, uma vez que possuem alguma doença crônica, alterações fisiológicas (imunodepressão, por exemplo) e desnutrição ${ }^{(13,14)}$. Um estudo de coorte realizado em 8 hospitais localizados nos estados de Carolina do Norte e na Virgínia, Estados Unidos, mostrou que quando o paciente idoso é diagnosticado com ICS tem-se um aumento da mortalidade, aumento do tempo de internação (mais 10 dias), chegando a alcançar um valor de 43.000,00 dólares em gastos hospitalares adicionais ${ }^{(15)}$.

Referente à presença de comorbidades prévias, 59,1\% dos pacientes apresentavam alguma doença cardíaca. Além disso, obtiveram-se resultados estatisticamente significativos para os pacientes diagnosticados com ICS com patologia cardíaca de base e desfecho clínico des- favorável, em que $50 \%$ destes foram a óbito. Estes dados corroboram com um estudo realizado na Bélgica ${ }^{(13)}$, em que entre os 109 pacientes que apresentavam ICS, 80 $(73,4 \%)$ possuíam alguma doença cardiovascular. Além disso, um estudo(l) multicêntrico, realizado em diferentes UTIs no mundo, verificou que em pacientes com diagnóstico clínico de infecção, a insuficiência cardíaca foi um dos fatores de risco independentemente associado a um maior risco de óbito durante a internação hospitalar.

A internação clínica se destacou neste estudo quando comparado aos demais tipos de internação, o que pode ser um possível reflexo da faixa etária e/ou presença de uma ou mais comorbidades prévias dos indivíduos participantes deste estudo. Diante do exposto, este resultado vai ao encontro com outro estudo ${ }^{(16)}$, o qual demostra maior número de internação em UTI clínica, correspondendo 4.407 admissões de longa permanência. Relacionando estes dados com o tempo de permanência na UTI, a média 
deste estudo foi de 12 dias, diferentemente de outro(15), o qual obteve uma média de internação de 23 dias para os pacientes diagnosticados com ICS. Este tempo pode ser ainda maior quando a ICS é causada por algum microrganismo multirresistente, podendo chegar a 37,2 dias de internação( ${ }^{(6)}$. O tempo de permanência hospitalar está parcialmente relacionado com episódios de ICS, em que quanto maior o período de exposição do paciente crítico, maior será o risco de infecção nosocomial(15-17).

Dentre as ICS notificadas, a maioria dos casos, neste estudo, foi decorrente de infecção por Staphylococcus coagulase negativa ( $\mathrm{SCoN}$ ), microrganismo encontrado na microbiota da pele humana, mas que vêm sendo um importante patógeno relacionado as infecções nosocomiais $^{(18)}$. Este resultado corrobora com outro estudo ${ }^{(19)}, 0$ qual constatou que os $\mathrm{SCoN}$ foram as bactérias que causaram de maneira mais frequente as ICS nos pacientes estudados. Outro estudo(4), que também utilizou metodologia automatizada na realização de hemoculturas, isolou as seguintes bactérias Gram positivas: Staphylococcus aureus (52\%), SCoN (32\%), Enterococcus spp. (8\%) e Streptococcus pneumoniae (8\%); e Gram negativas: Escherichia coli $(40,9 \%)$, Klebsiella spp. (27,3\%), Pseudomonas aeruginosa (22,7\%), Citrobacter spp. (9,1\%).

Além de ser um dos principais agentes causais de ICS, os SCoN vêm apresentando cepas resistentes aos antimicrobianos, sobretudo aquelas que possuem resistência a meticilina ${ }^{(18)}$. A taxa de resistência detectada fenotipicamente neste estudo para MRSCoN vai ao encontro com a de outro estudo(20), que demostrou alta taxa de Staphylococcus resistente a meticilina, em que $70 \%$ dos isolados de Staphylococcus epidermidis foram resistentes ao antibiótico testado. O surgimento dessas cepas de SCoN resistentes complica ainda mais o quadro clínico do paciente e o tratamento de infecções, sendo necessário adotar novas terapias antimicrobianas, que atuem de maneira efetiva nas diferentes espécies das bactérias pertencentes ao grupo de $\mathrm{SCoN}{ }^{(18)}$.

Apesar do estudo ser realizado na UTI adulto de um único hospital, nota-se a relevância de pesquisar a ICS, a fim de conhecer o perfil dos agentes patogênicos responsáveis pelo processo infeccioso, bem como averiguar se a propagação bacteriana pode possuir relação com a contaminação cruzada do ambiente hospitalar e mãos dos profissionais de saúde. As limitações encontradas foram a dificuldade de encontrar dados referentes às variáveis pesquisadas nos prontuários eletrônicos, gerando exclusão de amostras e o curto período analisado. Ressaltase que é necessário realizar novos estudos, abrangendo mais UTIs adulto, o que permitirá identificar o perfil das ICS em hospitais da região, bem como os possíveis fatores de risco para o desenvolvimento da infecção, seja em relação as comorbidades prévias do paciente ou associado ao uso de dispositivos médicos invasivos.

\section{CONCLUSÃO}

Neste estudo podemos identificar que o sexo masculino e indivíduos idosos foram os mais acometidos com ICS na UTI. Além disso, constatou-se que a grande maioria dos pacientes com ICS sofriam de comorbidades prévias, com destaque para as doenças cardíacas. Pode-se identificar também que o SCoN foi o agente bacteriano mais envolvido com os casos de ICS na UTI estudada, em que destes, a maioria apresentava resistência à oxacilina. 


\section{REFERÊNCIAS}

I. Vincent JL, Rello J, Marshall J, et al. International study of the prevalence and outcomes of infection in intensive care units. JAMA 2009; 302(21):2323-2329. http://dx.doi. org/10.100I/jama.2009.1754

2. Silva E, Dalfior Junior L,Fernandes HS, et al. Prevalence and outcomes of infections in Brazilian ICUs: a subanalysis of EPIC II study. Rev Bras Ter Intensiva. 2012; 24(2): I43-I 50. http://dx.doi.org//0.1590/S0103-507X201 2000200008.

3. Rutkowska K, Przybyła M, Misiołek H. Healthcare-associated infections in a newly opened intensive care unit. Anaesthesiol Intensive Ther. 2013, 45(2):62-66.http://dx.doi. org/I0.5603/AIT.2013.0014

4. Vasudeva N, Nirwan PS, Shrivastava P. Bloodstream infections and antimicrobial sensitivity patterns in a tertiary care hospital of India. TherAdv Infect Dis. 2016; 3(5): I I9127. http://dx.doi.org/I0. I I77/2049936 I I6666983

5. Scerbo MH, Kaplan HB, Dua A, et al. Beyond Blood Culture and Gram Stain Analysis:A Review of Molecular Techniques for the Early Detection of Bacteremia in Surgical Patients. Surg Infect (Larchmt). 2016; 17(3):294-302. http://dx.doi. org/10.1089/sur.2015.099

6. Riu M, Chiarello P, Terradas R, et al. Incremental cost of nosocomial bacteremia according to the focus of infection and antibiotic sensitivity of the causative microorganism in a university hospital. Medicine 2017; 96(I7):e6645. http:// dx.doi.org/I0.1097/MD.0000000000006645.

7. Agência Nacional de Vigilância Sanitária (ANVISA).Programa Nacional de Prevenção e Controle de Infecções Relacionadas à Assistência à Saúde (2016-2020). Brasília, DF: ANVISA; 2016. Disponível em: <http://portal.anvisa.gov. br/documents/33852/3074 I 75/PNPCIRAS+2016-2020/ f3eb5d5I-6I6c-49fa-8003-0dcb8604e7d9>. Acesso em: 0 I mai. 2018

8. Cohen B, Choi YJ, Hyman S, et al. Gender differences in risk of bloodstream and surgical site infections. J Gen Intern Med. 2013; 28(10): I 3 18-25. http://dx.doi.org//0.1007/ s| |606-0|3-242|-5

9. Luft D, Schmoor C, Wilson C, et al. Central venous catheter-associated bloodstream infection and colonisation of insertion site and catheter tip. What are the rates and risk factors in haematology patients? Ann Hematol. 2010; 89(I2): I 265-75. http://dx.doi.org/10.1007/s00277$010-1005-2$

10. Silva ACSS, Santos El, Penha RS, et al. Evidências científicas brasileiras acerca da infecção primária da corrente sanguí- nea em pediatria. Rev Enf Atual. 2018; 82(20):62-70.

I I. Suzuki M, Satoh N, Nakamura M, et al. Bacteremia in hemodialysis patients. World J Nephrol. 2016; 5(6):489-496. http://dx.doi.org// 0.5527/wjn.v5.i6.489

12. Fram D, Okuno MF, Taminato M, et al. Risk factors for bloodstream infection in patients at a Brazilian hemodialysis center: a case-control study. BMC Infect Dis. 20I5; I5: 158. http://dx.doi.org/I0.I 186/s I 2879-015-0907-y

13. Reunes S, Rombaut V, Vogelaers D, et al. Risk factors and mortality for nosocomial bloodstream infections in elderly patients. Eur J Intern Med. 20 I I; 22(5):e39-44. http://dx.doi. org/I0.1016/j.ejim.20II.02.004

I4. Ma HY, Hung IC, Huang YH, et al. Prognostic factors of health care-associated bloodstream infection in adult patients $\geq 40$ years of age. Am J Infect Control.20 I8;46(I): I I I- I I 4 . http://dx.doi.org//0.1016/j.ajic.2017.07.003

15. Kaye KS, Marchaim D, Chen TY, et al. Effect of nosocomial bloodstream infections on mortality, length of stay, and hospital costs in older adults. J Am Geriatr Soc. 2014; 62(2):306- I I. http://dx.doi.org/ I0. I I I //jgs. I 2634

16. Alberti C, Brun-Buisson C, Burchardi H, et al. Epidemiology of sepsis and infection in ICU patients from an international multicentrecohorte study. Intensive Care Med. 2002; 28(2):|08-2I. http://dx.doi.org//10.1007/ s00134-00 I-I I43-z

17. Nelson RE, Samore MH, Jones M, et al. Reducing time-dependent bias in estimates of the attributable cost of health care-associated methicillin-resistant Staphylococcus aureus infections: a comparison of three estimation strategies. Med Care. 20I5; 53(9):827-34. http://dx.doi.org/10.1097/ MLR.0000000000000403

18. Becker K, Heilmann C, Peters G. Coagulase-negative staphylococci. Clin Microbiol Rev. 2014;2 7(4):870-926. http:// dx.doi.org/1 10.1 I28/CMR.00109-13

19. Kolonen A, Sinisalo M, Huttunen R, et al. Bloodstream infections in acute myeloid leukemia patients treated according to the Finnish Leukemia Group AML-2003 protocol - a prospective nationwide study. Infect Dis (Lond). 2017; 49:799-808. http://dx.doi.org// 0.1080/23744235.2 017.1347814

20. Hashem AA, Abd El Fadeal NM, Shehata AS. In vitro activities of vancomycin and linezolid against biofilm-producing methicillin-resistant staphylococci species isolated from catheter-related bloodstream infections from an Egyptian tertiary hospital. J Med Microbiol. 2017; 66(6):744-52. http://dx.doi.org/10.1099/jmm.0.000490 